\title{
Parâmetros nutricionais de indivíduos com COVID-19 hospitalizados em UTI
}

\author{
Nutritional parameters of individuals with COVID-19 hospitalized in ICU \\ Parámetros nutricionales de personas con COVID-19 hospitalizadas en UCI
}

Recebido: 05/09/2021 | Revisado: 12/09/2021 | Aceito: 16/09/2021 | Publicado: 18/09/2021

\author{
Gabriella Ribeiro Ernesto do Rêgo \\ ORCID: https://orcid.org/0000-0002-7520-6439 \\ Faculdades Integradas de Patos, Brasil \\ E-mail: gaby_ribeiroo@hotmail.com \\ Lucas de Almeida Moura \\ ORCID: https://orcid.org/0000-0002-4927-4980 \\ Universidade Federal da Paraíba, Brasil \\ E-mail: moura.a.lucas@gmail.com
}

\begin{abstract}
Resumo
Por se tratar de uma doença que afeta principalmente o sistema respiratório, a COVID-19 pode evoluir para uma situação mais crítica, levando até mesmo à falência de múltiplos órgãos, chegando a ser fatal. Quadros nos quais complicações respiratórias agudas passam a requerer permanência prolongada em Unidades de Terapia Intensiva (UTIs) são as principais causas de morbidade e mortalidade em pacientes com COVID-19. A permanência em UTI, sobretudo em tempo prolongado, é, por si só, uma causa da alteração de parâmetros nutricionais. Diante disto, o presente trabalho tem como objetivo analisar o comportamento do estado e parâmetros nutricionais de pacientes com COVID-19 admitidos em UTI durante o curso da pandemia. Foi realizada uma revisão integrativa da literatura, na qual foi utilizado método sistematizado para síntese e análise dos trabalhos científicos disponíveis. Verificou-se que, nos casos de pacientes COVID-19 agravados, em tratamento em UTI, constatou-se significativa presença de comorbidades como diabetes, hipertensão e associação destas entre os indivíduos hospitalizados, bem como mudanças no perfil de ingestão calórica e proteica e no balanço nitrogenado. Também foi constatado que desnutrição e moderado a alto risco nutricional foram considerados fatores de risco para maiores períodos de internação hospitalar. A possibilidade de investigação de parâmetros mais minuciosos é travessada pelo próprio contexto pandêmico, no qual a urgência no manejo dos pacientes e a necessidade de isolamento e adoção de protocolos sanitários muitas vezes impossibilita a realização das pesquisas científicas com pacientes críticos em decorrência da COVID-19.
\end{abstract}

Palavras-chave: Infecção por coronavírus; UTI; Estado nutricional; COVID-19; Pandemia.

\begin{abstract}
As it is a disease that mainly affects the respiratory system, COVID-19 can evolve into a more critical situation, even leading to multiple organ failure, even fatal. Conditions in which acute respiratory complications require prolonged stay in Intensive Care Units (ICUs) are the main causes of morbidity and mortality in patients with COVID-19. The stay in the ICU, especially for a long time, is, by itself, a cause of changes in nutritional parameters. In view of this, this study aims to analyze the behavior of the nutritional status and parameters of patients with COVID-19 admitted to the ICU during the course of the pandemic. An integrative literature review was carried out, in which a systematic method was used for the synthesis and analysis of available scientific papers. It was found that, in cases of aggravated COVID-19 patients undergoing treatment in the ICU, there was a significant presence of comorbidities such as diabetes, hypertension and their association among hospitalized individuals, as well as changes in the profile of caloric and protein intake and in the nitrogen balance. It was also found that malnutrition and moderate to high nutritional risk were considered risk factors for longer hospital stays. The possibility of investigating more detailed parameters is crossed by the pandemic context itself, in which the urgency of patient management and the need for isolation and adoption of sanitary protocols often make it impossible to carry out scientific research with critically ill patients due to COVID-19. Keywords: Coronavirus infection; ICU; Nutritional status; COVID-19; Pandemic.

\section{Resumen}

Al tratarse de una enfermedad que afecta principalmente al sistema respiratorio, el COVID-19 puede evolucionar hacia una situación más crítica, llegando incluso a producir fallos multiorgánicos, incluso mortales. Las condiciones en las que las complicaciones respiratorias agudas requieren estancia prolongada en Unidades de Cuidados Intensivos (UCI) son las principales causas de morbimortalidad en pacientes con COVID-19. La estancia en la UCI, especialmente durante un tiempo prolongado, es, por sí misma, una causa de cambios en los parámetros nutricionales. Ante esto, este estudio tiene como objetivo analizar el comportamiento del estado y parámetros nutricionales de los pacientes con COVID-19 ingresados en la UCI durante el transcurso de la pandemia. Se realizó una revisión integradora de la literatura, en la que se utilizó un método sistemático para la síntesis y análisis de los artículos científicos disponibles.
\end{abstract}


Se encontró que, en los casos de pacientes con agravamiento del COVID-19 en tratamiento en la UCI, existía una presencia significativa de comorbilidades como diabetes, hipertensión y su asociación entre los individuos hospitalizados, así como cambios en el perfil de ingesta calórica y proteica. y en el balance de nitrógeno. También se encontró que la desnutrición y el riesgo nutricional moderado a alto se consideraron factores de riesgo para estadías hospitalarias más prolongadas. La posibilidad de investigar parámetros más detallados es atravesada por el propio contexto pandémico, en el que la urgencia del manejo del paciente y la necesidad de aislamiento y adopción de protocolos sanitarios muchas veces imposibilitan la realización de investigación científica con pacientes críticos por COVID-19.

Palabras clave: Infección por coronavirus; UCI; Estados nutricionales; COVID-19; Pandemia.

\section{Introdução}

O surgimento da pandemia de COVID-19 passou a apresentar desafios e ameaças sem precedentes para a população e para os sistemas de saúde em todo o mundo. Por se tratar de uma doença que afeta principalmente o sistema respiratório, ela pode evoluir para uma situação mais crítica, levando até mesmo à falência de múltiplos órgãos, chegando a ser fatal (Zhu et al., 2020; Chen et al., 2020; Huang et al., 2020; Bouadma et al., 2020; Zhou et al., 2020).

Trata-se de uma doença ocasionada por infecção viral pelo SARS-CoV-2 de rápida disseminação, resultando em doença crítica prolongada ou morte em diversos pacientes. Com o número crescente de casos e mortes relatado diariamente, a COVID19 tornou-se uma das mais importantes crises de saúde pública na história moderna (Micic et al., 2020).

Nesse sentido, quadros nos quais complicações respiratórias agudas passam a requerer permanência prolongada em Unidades de Terapia Intensiva (UTIs) são as principais causas de morbidade e mortalidade em pacientes com COVID-19, sendo mais agravados quando estes são indivíduos idosos e com mais de uma comorbidade prévia, o que decorre em piores resultados e maior mortalidade (Zhu et al., 2020; Chen et al., 2020; Huang et al., 2020; Bouadma et al., 2020; Zhou et al., 2020).

A permanência em UTI, sobretudo em tempo prolongado, é também, por si só, uma causa bastante elucidada de desnutrição, com perda de massa magra e função esquelética na maior parte dos casos o que, por sua vez, pode levar a uma baixa qualidade de vida futura, incapacidades e morbidades mesmo muito tempo após a alta da terapia (Singer et al., 2019). Além disso, quando da presença de infecção pelo SARS-CoV-2, a inflamação e o desenvolvimento de sepse podem contribuir ainda mais para o aumento de todas as alterações já citadas. Logo, a realização de um tratamento e avaliação nutricional adequados também são bastante elucidados como maneira de reduzir efetivamente estas complicações e melhorar os resultados clínicos em diversas condições, sobretudo nos casos de internação em UTI (Gomes et al., 2018; Singer et al., 2019; Volkert et al., 2019; Barazzoni et al., 2020).

Diante disto, compreende-se que os parâmetros nutricionais dos indivíduos acometidos pela COVID-19 devem ser considerados, especialmente quando do manejo destes em Unidades de Terapia Intensiva. Assim, por meio de uma revisão integrativa da literatura, o presente trabalho tem como objetivo analisar o comportamento do estado e parâmetros nutricionais de pacientes com COVID-19 admitidos em UTI durante o curso da pandemia.

\section{Metodologia}

Trata-se de uma revisão integrativa da literatura, na qual foi utilizado método sistematizado para síntese e análise dos trabalhos científicos disponíveis. Seguiu-se a metodologia proposta por Souza, Silva e Carvalho (2010) que contempla os seguintes passos: definição da questão norteadora; elaboração da estratégia e execução de busca; seleção dos estudos encontrados; escolha das informações relevantes nos estudos selecionados; análise crítica dos estudos incluídos; discussão dos resultados; e apresentação da revisão integrativa.

Foi definida como questão norteadora a seguinte pergunta: "Como se comportam os parâmetros nutricionais diante de quadros de COVID-19 de pacientes hospitalizados em UTI?". A pesquisa foi realizada em julho de 2021 através do portal online 
da Biblioteca Virtual em Saúde (BVS), na qual foram utilizados os descritores combinados ao operador booleano AND: (COVID-19) AND (nutritional therapy) AND (intensive care unit).

Foram definidos elegíveis os artigos originais, com abordagem quantitativa ou qualitativa, entre dezembro de 2019 e junho de 2021, disponíveis gratuitamente em texto completo, nos idiomas inglês, português ou espanhol e que contemplassem a questão norteadora proposta. Artigos de revisão e/ou meta análises não foram incluídos. A escolha do período considerou a identificação do primeiro caso de infecção pelo SARS-CoV-2 no mundo, em Wuhan, na China.

Os artigos foram selecionados por meio da leitura inicial dos títulos e resumos, o que possibilitou a exclusão de trabalhos duplicados e aqueles que não se enquadravam na temática e nos critérios de elegibilidade previamente definidos. Após isto, os artigos restantes foram lidos na íntegra e incluídos no estudo.

Considerando a novidade do assunto referente à pandemia de COVID-19 e as dificuldades para a realização de pesquisas científicas durante este período, que requer isolamento social, entende-se que a limitação da quantidade de trabalhos encontrados é justificada por este contexto.

Os dados extraídos dos artigos selecionados incluíram: título, autor, objetivos, metodologia, resultados (caso possuíssem) e conclusões de cada estudo, sendo que os principais dados foram sistematizados e apresentados em forma de quadro na seção dos resultados. Um fluxograma ilustra o processo de revisão aplicado, detalhando a quantidade de trabalhos encontrado e/ou selecionados a partir da realização de cada etapa da metodologia empregada nesse trabalho.

\section{Resultados e Discussão}

A Figura 1 ilustra o procedimento metodológico adotado para a revisão integrativa realizada. Após o processo de verificação de artigos duplicados e posterior leitura dos títulos e resumos, dos 50 estudos encontrados, que em sua totalidade foram oriundos da base de dados MEDLINE, 8 estudos foram incluídos nessa revisão atendendo aos critérios de inclusão préestabelecidos, bem como pela abordagem à questão norteadora proposta.

Figura 1 - Fluxograma de revisão e seleção dos artigos da pesquisa.

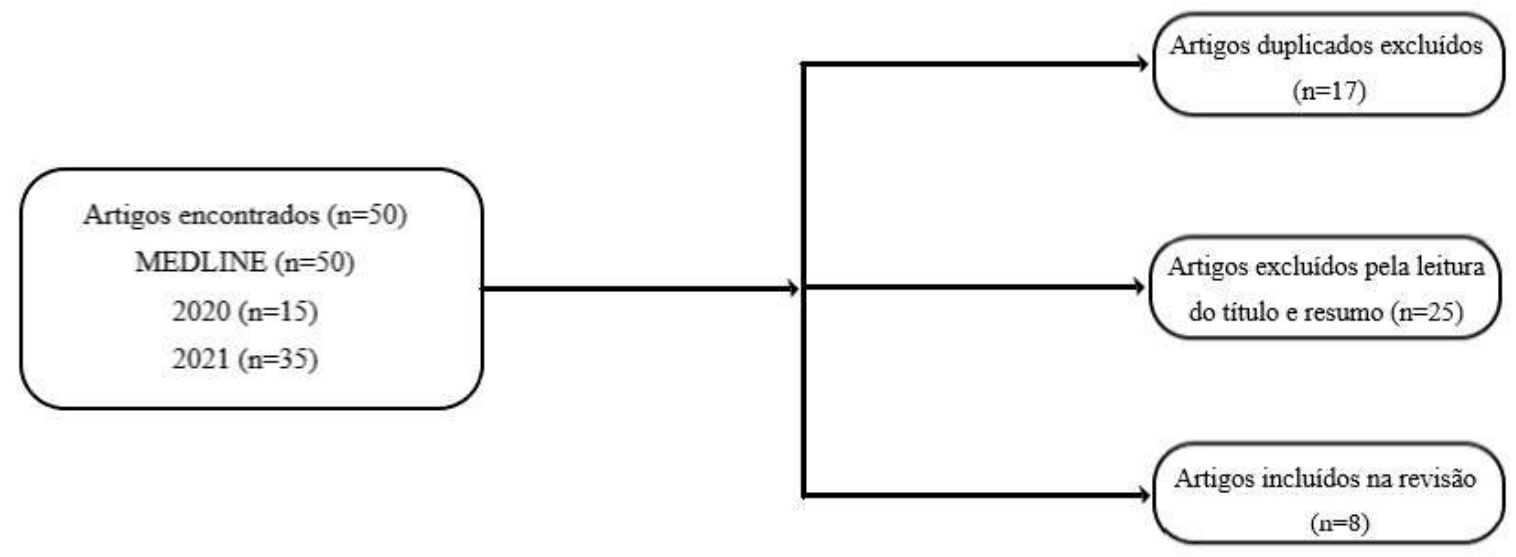

Fonte: Autores (2021).

Ao ser considerado o período em que a pandemia foi reconhecida internacionalmente pela Organização Mundial da Saúde (OMS), em 30 de janeiro de 2020, como uma situação de emergência em saúde pública de interesse internacional (ESPIN), chama-se atenção para o fato de que a disponibilidade de estudos sobre a COVID-19 desde então passou a ganhar diversos direcionamentos e, no caso da associação dos estudos que possuem como principal objeto de investigação a associação entre a 
doença e os parâmetros nutricionais de indivíduos, estes ainda não puderam ser largamente produzidos, uma vez que a própria situação emergencial, que exige isolamento e distanciamento social, bem como diversas outras medidas de higiene, por si só, impedem que os pesquisadores possam realizar pesquisas mais substanciais sobre o tema.

O Quadro 1 apresenta a caracterização dos estudos selecionados nessa pesquisa. A maior parte dos estudos seguiram delineamento observacional. Destes, a maioria $(n=5)$ se refere a pesquisas de coorte retrospectiva e ambidirecional, com dados de adultos hospitalizados durante um período da pandemia de COVID-19 (Formenti et al., 2021; Osuna-Padilla et al., 2021; Larrazabal Jr. et al., 2021; Wu et al., 2021; Lin et al., 2021). Também foi verificada a realização de estudo de intervenção $(\mathrm{n}=$ 1) (Watteville et al., 2020) e estudos longitudinais observacionais $(n=2)$ (Bedock et al., 2021; Nicolau et al., 2021).

Quadro 1 - Estudos encontrados e selecionados através da pesquisa de revisão.

\begin{tabular}{|c|c|c|c|c|}
\hline Título & Autores(as) & Objetivo geral & Método & Conclusões \\
\hline $\begin{array}{l}\text { Evolution of } \\
\text { Nutritional } \\
\text { Status after } \\
\text { Early } \\
\text { Nutritional } \\
\text { Managemen } \\
\text { t in COVID- } \\
19 \\
\text { Hospitalized } \\
\text { Patients }\end{array}$ & Bedock et al. & $\begin{array}{l}\text { Descrever a evolução } \\
\text { dos parâmetros nutricionais en } \\
\text { tre a admissão e } 30 \text { dias após } \\
\text { a alta hospitalar e determinar } \\
\text { os fatores preditivos de mau } \\
\text { resultado nutricional após a } \\
\text { recuperação } \\
\text { em pacientes adultos } \\
\text { com COVID-19. }\end{array}$ & $\begin{array}{l}\text { Estudo longitudinal observacional no } \\
\text { qual foram relatados os resultados } \\
\text { após a alta em 91 de } \\
114 \text { pacientes inicialmente admitidos } \\
\text { para COVID-19 que receberam } \\
\text { tratamento nutricional precoce. O } \\
\text { estado nutricional foi definido pelos } \\
\text { critérios do GLIM e comparado entre a } \\
\text { admissão e o } 30^{\circ} \text { dia após a alta. Os } \\
\text { preditores de linha de base do estado } \\
\text { nutricional no dia } 30 \text { foram avaliados } \\
\text { por meio de regressão logística. }\end{array}$ & $\begin{array}{lr}\text { Com o manejo } & \text { nutricional } \\
\text { precoce, a } & \text { maioria } \\
\text { dos pacientes hospitalizados por } & \text { melhorou } \\
\text { COVID-19 r n } & \text { maricionais após } \\
\text { os parâmetros nutricion } \\
\text { a alta. Estes resultados reforçam } \\
\text { a importância de cuidados } \\
\text { nutricionais em COVID- } \\
19 \text { pacientes internados } \\
\text { em medicina departamentos, } \\
\text { especialmente naqueles } \\
\text { transferidos da UTI. }\end{array}$ \\
\hline $\begin{array}{l}\text { Influence of } \\
\text { nutritional } \\
\text { status on } \\
\text { clinical } \\
\text { outcomes } \\
\text { among } \\
\text { hospitalized } \\
\text { patients } \\
\text { with } \\
\text { COVID-19 }\end{array}$ & Nicolau et al. & $\begin{array}{l}\text { Avaliar se a presença } \\
\text { de desnutrição entre pacientes } \\
\text { internados por COVID-19 teve } \\
\text { algum impacto nos desfechos } \\
\text { clínicos em comparação } \\
\text { com pacientes com a mesma } \\
\text { condição, mas bem nutridos. }\end{array}$ & $\begin{array}{l}\text { Estudo transversal com } 75 \text { pacientes } \\
\text { hospitalizados em decorrência da } \\
\text { Covid-19. Foi realizada Avaliação } \\
\text { Subjetiva Global (ASG) por telefone e } \\
\text { os parâmetros clínicos foram extraídos } \\
\text { do prontuário eletrônico. }\end{array}$ & $\begin{array}{l}\text { A presença de mau estado } \\
\text { nutricional está relacionada a } \\
\text { maior tempo de internação, } \\
\text { maior internação em UTI e } \\
\text { maior mortalidade. }\end{array}$ \\
\hline $\begin{array}{l}\text { Early } \\
\text { enteral } \\
\text { nutrition } \\
\text { improves } \\
\text { the outcome } \\
\text { of critically } \\
\text { ill patients } \\
\text { with } \\
\text { COVID-19: } \\
\text { A } \\
\text { retrospectiv } \\
\text { e study }\end{array}$ & Wu et al. & $\begin{array}{lr}\text { Avaliar } & \text { o estado } \\
\text { nutricional de pacientes crítico } \\
\text { s de COVID-19 e determinar } \\
\text { qual a via } \quad \text { a via } \\
\text { de suporte nutricional mais } \\
\text { vantajosa. }\end{array}$ & 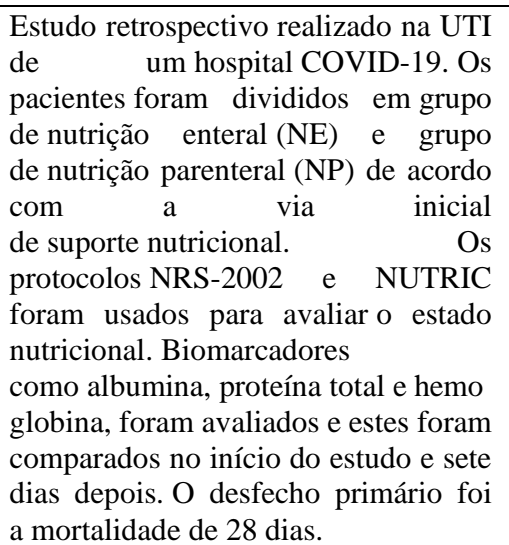 & $\begin{array}{l}\text { A incidência de risco nutricional } \\
\text { em pacientes criticamente } \\
\text { enfermos com COVID-19 é } \\
\text { muito alta. NE precoce pode ser } \\
\text { benéfico para os resultados } \\
\text { do paciente. }\end{array}$ \\
\hline $\begin{array}{l}\text { Nutritional } \\
\text { practice in } \\
\text { critically ill } \\
\text { COVID-19 } \\
\text { patients: A } \\
\text { multicenter } \\
\text { ambidirectio } \\
\text { nal cohort } \\
\text { study in } \\
\text { Wuhan and } \\
\text { Jingzhou }\end{array}$ & Lin et al. & $\begin{array}{l}\text { Descrever as características } \\
\text { clínicas e práticas nutricionais } \\
\text { em uma coorte de pacientes } \\
\text { críticos em decorrência } \\
\text { da COVID-19. }\end{array}$ & $\begin{array}{l}\text { Estudo de coorte multicêntrico } \\
\text { e ambidirecional conduzido em } \\
11 \text { hospitais na província de Hubei, na } \\
\text { China. Todos os pacientes críticos de } \\
\text { COVID-19 elegíveis das unidades de } \\
\text { cuidados intensivos do hospital em } 6 \\
\text { de Março de } 2020 \text { foram incluídos. A } \\
\text { coleta de dados foi realizada por meio } \\
\text { de formulários de relato de caso. }\end{array}$ & $\begin{array}{l}\text { O estudo mostrou que a Nutrição } \\
\text { Enteral foi frequentemente } \\
\text { aplicada em pacientes críticos } \\
\text { com } \\
\text { fornecimento de energia pode ser } \\
\text { abaixo do ideal neste estudo, } \\
\text { exigindo mais atenção. }\end{array}$ \\
\hline
\end{tabular}




\begin{tabular}{|c|c|c|c|c|}
\hline $\begin{array}{l}\text { Outcomes } \\
\text { of } \\
\text { nutritionally } \\
\text { at-risk } \\
\text { Coronavirus } \\
\text { Disease } \\
2019 \\
\text { (COVID 19) } \\
\text { patients } \\
\text { admitted in } \\
\text { a tertiary } \\
\text { government } \\
\text { hospital: A } \\
\text { follow-up } \\
\text { study of the } \\
\text { MalnutriCo } \\
\text { V study }\end{array}$ & $\begin{array}{l}\text { Larrazabal; } \\
\text { Chiu; Palileo- } \\
\text { Villanueva }\end{array}$ & $\begin{array}{l}\text { Determinar os resultados } \\
\text { de pacientes adultos } \\
\text { com COVID-19 internados em } \\
\text { um hospital privado } \\
\text { terceirizado pelo governo } \\
\text { segundo parâmetros de risco } \\
\text { e o estado nutricional. }\end{array}$ & $\begin{array}{l}\text { Tratou-se de um estudo do tipo } \\
\text { retrospectivo com pacientes adultos C } \\
\text { OVID-19 hospitalizados que foram } \\
\text { selecionados usando a ferramenta do } \\
\text { grau de avaliação subjetiva global, } \\
\text { modificada da Sociedade Filipina } \\
\text { de Nutrição Enteral e Parenteral. }\end{array}$ & $\begin{array}{l}\text { A transferência para UTI foi o } \\
\text { único fator de risco demonstrado } \\
\text { no estudo. Foram fatores de risco } \\
\text { para longos períodos de } \\
\text { internação: } \\
\text { desnutrição, risco nutricional } \\
\text { moderado e } \\
\text { alto risco nutricional, enquanto } \\
\text { apenas a desnutrição foi o fator } \\
\text { de risco para a menor } \\
\text { probabilidade de receber } \\
\text { alta. Reiterou-se que a avaliação } \\
\text { e o suporte nutricional são } \\
\text { importantes para mitigar os } \\
\text { efeitos do COVID-19. }\end{array}$ \\
\hline $\begin{array}{l}\text { High } \\
\text { nutritional } \\
\text { risk using } \\
\text { NUTRIC- } \\
\text { Score is } \\
\text { associated } \\
\text { with worse } \\
\text { outcomes in } \\
\text { COVID-19 } \\
\text { critically ill } \\
\text { patients }\end{array}$ & $\begin{array}{l}\text { Osuna-Padilla } \\
\text { et al. }\end{array}$ & $\begin{array}{l}\text { Descrever } \\
\text { a associação entre risco nutrici } \\
\text { onal e tempo de internação, } \\
\text { dias em ventilação } \\
\text { mecânica e mortalidade } \\
\text { hospitalar em pacientes infecta } \\
\text { dos pelo SARS-CoV-2. }\end{array}$ & $\begin{array}{l}\text { Estudo de coorte retrospectiva } \\
\text { com pacientes críticos em ventilação } \\
\text { mecânica. Avaliou- } \\
\text { se o risco nutricional no início do } \\
\text { estudo usando a pontuação } \\
\text { NUTRIC. Modelos de regressão linear } \\
\text { e logística foram utilizados para } \\
\text { analisar a associação entre a } \\
\text { pontuação NUTRIC e os resultados } \\
\text { clínicos (dias em ventilação } \\
\text { mecânica, tempo de permanência no } \\
\text { hospital, bem como a mortalidade } \\
\text { hospitalar). Uma análise } \\
\text { sobrevivência foi realizada usando } \\
\text { curvas de Kaplan-Meier. }\end{array}$ & $\begin{array}{l}\text { O alto risco nutricional está } \\
\text { relacionado } \\
\text { à mortalidade em pacientes criti } \\
\text { camente enfermos com SARS- } \\
\text { CoV-2. Or sobrepeso e } \\
\text { a obesidade são comuns na } \\
\text { amostra analisada. Mais estudos } \\
\text { são necessários para elucidar o } \\
\text { impacto } \\
\text { da terapia nutricional no curso e } \\
\text { resultados da infecção. }\end{array}$ \\
\hline $\begin{array}{l}\text { Easy-to- } \\
\text { prescribe } \\
\text { nutrition } \\
\text { support in } \\
\text { the intensive } \\
\text { care in the } \\
\text { era of } \\
\text { COVID-19. }\end{array}$ & $\begin{array}{l}\text { Watteville et } \\
\text { al. }\end{array}$ & $\begin{array}{l}\text { Produzir um protocolo simples } \\
\text { e fácil de prescrição nutricional } \\
\text { e avaliar sua qualidade a partir } \\
\text { da utilização destes por } \\
\text { médicos envolvidos no } \\
\text { cuidado de pacientes Covid-19 } \\
\text { em UTI. }\end{array}$ & $\begin{array}{l}\text { Um protocolo simplificado de } \\
\text { terapia nutricional foi distribuído a } \\
\text { todos os médicos }(\mathrm{n}=122) \text { de uma } \\
\text { equipe médica de UTI durante } \\
\text { a pandemia de COVID- } \\
\text { 19. Os nutricionistas clínicos estimara } \\
\mathrm{m} \text { as metas de energia para as fases } \\
\text { aguda e pós-aguda na admissão } \\
\text { do paciente e sugeriram adaptações } \\
\text { da terapia nutricional. As situações } \\
\text { mais complexas foram discutidas } \\
\text { com nutricionistas clínicos e, quando } \\
\text { necessário, uma avaliação clínica foi } \\
\text { realizada. Para facilitar ainda mais } \\
\text { o processo, um gráfico } \\
\text { com estimativas de prescrição também } \\
\text { foi distribuído para toda a equipe da } \\
\text { UTI médica. No final da } \\
\text { onda pandêmica um questionário } \\
\text { com } 13 \text { itens foi enviado por e-mail à } \\
\text { equipe médica da UTI para obter } \\
\text { sua opinião sobre a terapia nutricional } \\
\text { sugerida. }\end{array}$ & $\begin{array}{l}\text { Nutricional terapia em criticame } \\
\text { nte doentes COVID- } \\
19 \text { pacientes é um desafio e a } \\
\text { aplicação específica do } \\
\text { presente pandemia protocolo } \\
\text { simplificado nutricional foi } \\
\text { avaliada como útil por uma } \\
\text { grande maioria } \\
\text { dos médicos. Pragmáticas e } \\
\text { simplificados protocolos são } \\
\text { úteis para assegurar } \\
\text { a qualidade de } \\
\text { nutricional terapia e pode ser } \\
\text { usado em futuros estudos para } \\
\text { avaliar o seu impacto real sobre } \\
\text { os resultados clínicos de } \\
\text { COVID-19 pacientes. }\end{array}$ \\
\hline $\begin{array}{l}\text { Nutrition } \\
\text { Support in } \\
\text { Patients } \\
\text { With Acute } \\
\text { Respiratory } \\
\text { Distress } \\
\text { Syndrome } \\
\text { COVID-19 }\end{array}$ & Formenti et al. & 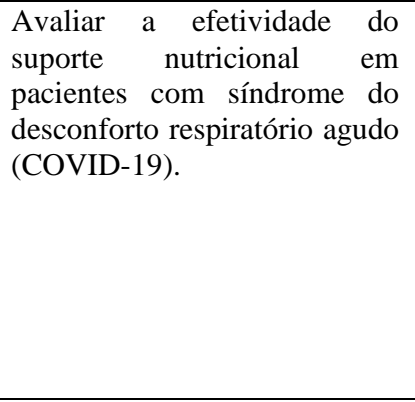 & $\begin{array}{l}\text { Estudo retrospectivo realizado na UTI } \\
\text { de um hospital universitário dedicado } \\
\text { ao atendimento de pacientes com } \\
\text { diagnóstico confirmado de COVID- } \\
19 \text {. Foram coletados dados de } 60 \\
\text { pacientes entre o período de fevereiro } \\
\text { e abril de } 2020 \text {, na admissão à UTI e } \\
\text { após } 3 \text { e } 7 \text { dias. A ingestão calórica e } \\
\text { proteica foi fornecida de acordo com } \\
\text { as recomendações europeias. }\end{array}$ & $\begin{array}{l}\text { Considerando um aumento da } \\
\text { resposta inflamatória na } \\
\text { admissão hospitalar, o protocolo } \\
\text { de nutrição provavelmente } \\
\text { precisa de ajustes para direcionar } \\
\text { a inflamação. Ainda precisa ser } \\
\text { comprovado se as necessidades } \\
\text { nutricionais e metabólicas de } \\
\text { pacientes ventilados com SDRA } \\
\text { COVID-19 são semelhantes às } \\
\text { de pacientes com SDRA. }\end{array}$ \\
\hline
\end{tabular}


A utilização do escore de pontuação NUTRIC (Nutrition Risk in the Critically Ill Score) foi verificada em três estudos (Osuna-Padilla et al., 2021; Wu et al., 2021; Lin et al., 2021). O NUTRIC é um escore de risco nutricional para pacientes críticos desenvolvido e validado, especificamente, para pacientes em Unidades de Terapia Intensiva (UTI). Ele combina parâmetros de pré-hospitalização como IMC, fome aguda e crônica e duração da admissão pré-hospitalar, com parâmetros inflamatórios agudos, como Interleucina-6, e crônicos (número de comorbidades) e gravidade da doença (escores APACHE-II e SOFA) em admissão na UTI, para avaliação do risco nutricional e desfechos associados, como o tempo de ventilação e mortalidade (Vries et al., 2018).

A avaliação subjetiva global (ASG), que é um método de avaliação do estado nutricional que se utiliza da combinação de diferentes fatores como perda de peso, exame físico do paciente, sintomas gastrintestinais, alterações funcionais e na ingestão alimentar (Gonzalez et al., 2010) foi utilizada em duas das pesquisas incluídas neste estudo (Larrazabal JR. et al., 2021; Nicolau et al., 2021). Além dela, a utilização de uma ferramenta mais recente, de diagnóstico de desnutrição, desenvolvida pela iniciativa Global Leadership on Malnutrition (GLIM) também foi verificada (Bedock et al., 2021). A GLIM envolveu várias sociedades de nutrição clínica, com alcance global, para se concentrar na padronização da prática clínica de diagnóstico da desnutrição (Cederholm et al., 2019). Em decorrência das restrições impostas pela pandemia, a utilização de métodos padronizados e validados para a avaliação de parâmetros e do estado nutricional, possíveis de serem aplicados através da tecnologia, como aplicativos, softwares e questionários online foi e tem sido frequente nos estudos com esta finalidade.

Quanto aos objetivos propostos, todos os oito trabalhos selecionados avaliaram parâmetros nutricionais de pacientes hospitalizados, incluindo aqueles em unidades de terapia intensiva (UTI), considerando a infecção pelo SARS-CoV-2, bem como a presença de comorbidades, necessidade de ventilação mecânica e o tipo de terapia nutricional empregada. Destaca-se o estudo de Watteville et al. (2020) que promoveu e avaliou a utilização de um protocolo de manejo nutricional para pacientes com COVID-19 hospitalizados em UTI de um hospital universitário de Genebra. O protocolo foi disponibilizado para a equipe médica do hospital para que esta pudesse aplicá-lo no atendimento aos pacientes infectados pelo SARS-CoV-2 e, posteriormente, por meio de questionário online, estes profissionais avaliaram a utilidade e aplicabilidade do protocolo.

Em relação aos resultados, verificou-se que, no caso da proposição de um protocolo de intervenção para manejo nutricional dos parâmetros nutricionais dos pacientes, este demonstrou ser bem aceito pela equipe médica, sobretudo pelos médicos da UTI, demonstrando sua utilidade e aplicabilidade. A abordagem adotada teve como principais objetivos simplificar o manejo nutricional no contexto de carga de trabalho e falta de especialização em nutrição clínica para profissionais indicados para a equipe médica da UTI; manutenção da qualidade da terapia nutricional, evitando-se tanto a subalimentação quanto a superalimentação; e evitar as principais complicações relacionadas à nutrição enteral, principalmente a pneumonia por aspiração (Watteville et al., 2020). Os pacientes com COVID-19, frequentemente demonstram baixa tolerância a nutrição enteral, uma vez que nos quadros críticos é comum apresentar alterações na motilidade intestinal, íleo paralítico e elevação dos resíduos gástricos (Arkin et al., 2020). Nesse sentido, Watteville et al. (2020) chamam a atenção para a necessidade de encontrar um equilíbrio entre atender às demandas nutricionais do paciente e evitar os riscos associados à tolerância intestinal, o que continua sendo um desafio.

Quanto aos demais estudos, de modo geral, verificou-se que nos casos de pacientes COVID-19 agravados, em tratamento em unidades de terapia intensiva, constatou-se significativa presença de comorbidades como diabetes, hipertensão e associação destas entre os indivíduos hospitalizados, bem como mudanças no perfil de ingestão calórica e proteica e no balanço nitrogenado (negativo (nas semanas iniciais de pacientes críticos)). Além disso, através da aplicação dos escores de risco nutricional mencionados anteriormente, também foi constatado que desnutrição e moderado a alto risco nutricional foram considerados fatores de risco para maiores períodos de internação hospitalar, com maior chance de mortalidade por agravamento do quadro quando da necessidade de transferência para UTI. 
Também foi verificado que, após a alta, cerca de 1/4 dos pacientes que foram internados em decorrência da COVID-19 apresentaram algum grau de desnutrição e isto apresentou diferenças quando das transferências destes pacientes para a UTI. A necessidade de oxigenoterapia acima de 5L/min. também demonstrou ser um preditor de desnutrição no pós-tratamento. As taxas de mortalidade foram maiores entre indivíduos com qualquer grau de desnutrição em comparação com pacientes bem nutridos.

Foi demonstrado também que a nutrição enteral (NE) e nutrição parenteral (NP) foi frequentemente aplicada a pacientes críticos com COVID-19, demonstrando-se eficientes para que os indivíduos pudessem atingir a necessidade diária de energia, o que é louvável uma vez que, como fora mencionado, a desnutrição demonstrou possuir relação com o maior tempo de internação hospitalar e menor probabilidade de alta, sobretudo para pacientes em UTI.

\section{Conclusão}

A pandemia de COVID-19 impactou o mundo de diversas formas e o modo de vida da população em todos os continentes e expôs os riscos de infecção e mortalidade que determinados grupos de indivíduos passaram a correr diante do cenário catastrófico.

A maior parte dos estudos avaliados revelou maior ocorrência de necessidade de internação para aqueles pacientes com COVID-19 que possuíam uma ou mais comorbidades prévias, com maior chance de agravamento e necessidade de transferência para unidade de terapia intensiva. Além disso, também foi verificada maior demanda de macro e micronutrientes nestes pacientes e mensuração de risco nutricional moderado a alto a partir dos escores empregados, bem como maior ocorrência de desnutrição em pacientes no pós-tratamento, sobretudo naqueles que necessitaram de alta carga de oxigenoterapia e permanência em UTI.

Enfatiza-se que a possibilidade de investigação de parâmetros mais minuciosos é atravessada pelo próprio contexto pandêmico, no qual a urgência no manejo dos pacientes e a necessidade de isolamento e adoção de protocolos sanitários muitas vezes impossibilita a realização das pesquisas científicas com pacientes críticos em decorrência da COVID-19.

Destaca-se, também, que pesquisas sobre essa temática ainda são escassas no Brasil, ressaltando-se a necessidade de realização de estudos longitudinais e/ou de coorte retrospectivos que avaliem o impacto da COVID-19 em parâmetros nutricionais de pacientes hospitalizados em decorrência da doença, especialmente àqueles nos quais os quadros incorrem para o agravamento, sendo necessária a internação em UTI. Estudos desse tipo podem embasar a adoção de protocolos de manejo nutricional para pacientes críticos com COVID-19, evitando morbidades e prejuízos decorrentes das doenças bem como a mortalidade.

\section{Referências}

Arkin, N., Krishnan, K., Chang, M. G. \& Bittner, E. A. (2020). Nutrition in critically ill patients with COVID-19: Challenges and special considerations. Clin. Nutr., 39(7), 2327-2328.

Barazzoni, R., Bischoff, S. C., Breda, J., Pirlich, M. \& Singer, P. (2020). ESPEN expert statements and practical guidance for nutritional management of individuals with SARS-CoV-2 infection. Clin. Nutr. ESPEN, 39, 1631-1638.

Bedock, D., Couffignal, J., Lassen, P. B. \& Soares, L. et al. (2021). Evolution of Nutritional Status after Early Nutritional Management in COVID-19 Hospitalized Patients. Nutrients, 13(7), 1-11.

Bouadma, L., Lescure, F. X., Lucet, J. C., Yazdanpanah, Y. \& Timsit, J. F. (2020). Severe SARS-CoV-2 infections: practical considerations and management strategy for intensivists. Intensive Care Medicine, 46, 579-582.

Cederholm, T., Jensen, G. L., Correia, M. I. T. D., Gonzalez, M. C. et al. (2019). GLIM criteria for the diagnosis of malnutrition - A consensus report from the global clinical nutrition Community. Journal of Cachexia, Sarcopenia and Muscle, 10(1), 207-217.

Chen, N., Zhou, M., Dong, X., Qu, J., Gong, F., Han, Y. et al. (2020). Epidemiological and clinical characteristics of 99 cases of 2019 novel coronavírus pneumonia in Wuhan, China: a descriptive study. The Lancet, 395, 507-513.

Formenti, P., Bichi, F., Castagna, V., Pozzi, T. \& Chiumello, D. (2021). Nutrition Support in Patients With Acute Respiratory Distress Syndrome COVID-19. Nutrition in Clinical Practice, 36(2), 500-501. 
Research, Society and Development, v. 10, n. 12, e196101220253, 2021

(CC BY 4.0) | ISSN 2525-3409 | DOI: http://dx.doi.org/10.33448/rsd-v10i12.20253

Gomes, F., Schuetz, P., Bounoure, L., Austin, P., Ballesteros-Pomar, M., Cederholm, T. et al. (2018). ESPEN guideline on nutritional support for polymorbid internal medicine patients. Clin. Nutr. ESPEN, 37, 336-353.

González, M. C., Borges, L. R., Silveira, D. H., Assunção, M. C. F. \& Orlandi, S. P. (2010). Validação da versão em português da avaliação subjetiva global produzida pelo paciente. Rev. Bras. Nutr. Clin., 25(2), 102-108.

Huang, C., Wang, Y., Li, X, Ren, L., Zhao, J., Hu, Y. et al. (2020). Clinical features of patients infected with 2019 novel coronavírus in Wuhan, China. The Lancet, 395, 497-500.

Larrazabal Jr., R. B., Chiu, H. H. C. \& Palileo-Villanueva, L. A. M. (2021). Outcomes of nutritionally at-risk Coronavirus Disease 2019 (COVID 19) patients admitted in a tertiary government hospital: A follow-up study of the MalnutriCoV study. Clin. Nutr. ESPEN, 43, 239-244.

Lin, J., Ke, L., Doig, G. S., Ye, B. et al. (2021). Nutritional practice in critically ill COVID-19 patients: A multicenter ambidirectional cohort study in Wuhan and Jingzhou. Asia Pac. J. Clin. Nutr., 30(1), 15-21.

Micic, D., Wall, E. \& Semrad, C. (2020). Nutrition support in the ICU - A refresher in the era of COVID-19. The American Journal of Gastroentrology, 115, $1367-1370$.

Nicolau, J., Ayala, L., Sanchís, P., Olivares, J. et al. (2021). Influence of nutritional status on clinical outcomes among hospitalized patients with COVID-19. Clin. Nutr. ESPEN, 43, 223-229.

Osuna-Padilla, I. A., Rodríguez-Moguel, N. C., Aguilar-Vargas, A. \& Rodríguez-Llamazares, S. (2021). High nutritional risk using NUTRIC-Score is associated with worse outcomes in COVID-19 critically ill patients. Nutrición Hospitalaria, 38(3), 540-544.

Singer, P., Blaser, A. R., Berger, M. M., Alhazzani, W., Calder, P. C., Casaer, M. P. et al. (2019). ESPEN guideline on clinical nutrition in the intensive care unit. Clin. Nutr. ESPEN, 38, 48-79.

Souza, M. T., Silva, M. D. \& Carvalho, R. (2010). Revisão Integrativa: o que é e como fazer. Einstein, 8(1), 102-106.

Volkert, D., Beck, A. M., Cederholm, T., Cruz-Jentoft, A., Goisser, S., Hooper, L. et al. (2019). ESPEN guideline on clinical nutrition and hydration in geriatrics. Clin. Nutr. ESPEN, 38, 10-47.

Vries, M. C. H., Koekkoek, W. A. C., Opdam, M. H., Blokland, D. V. \& Zanten, A. R. H. V. (2018). Nutritional assessment of critically ill patients: validation of the modified NUTRIC score. European Journal of Clinical Nutrition, 72, 428-435.

Watteville, A., Genton, L., Barcelos, G. K., Pugin, J., Pichard, C. \& Heidegger, C. P. (2020). Easy-to-prescribe nutrition support in the intensive care in the era of COVID-19. Clin. Nutr. ESPEN, 39, 74-78.

Wu, S., Lou, J., Xu, P., Luo, R. \& Li, L. (2021). Early enteral nutrition improves the outcome of critically ill patients with COVID-19: A retrospective study. Asia Pac. J. Clin. Nutr., 30(2), 192-198.

Zhou, F., Yu, T., Du, R., Fan, G., Liu, Y., Liu, Z. et al. (2020). Clinical course and risk factors for mortality of adult inpatients with COVID-19 in Wuhan, China: a retrospective cohort study. The Lancet, 395, 1054-1062.

Zhu, N., Zhang, D., Wang, W., Li, X., Yang, B., Song, J. et al. (2020). A novel coronavírus from patients with pneumonia in china, 2019. N. Engl. J. Med., 382, 727-733. 